\section{A portable fast neutron radiography system for non-destructive analysis of composite materials}

Erol Kam, Iskender A. Reyhancan, Recep Biyik

\begin{abstract}
Depending on the neutron energy used, neutron radiography can be generally categorized as fast and thermal neutron radiography. Fast neutron radiography (FNR) with neutron energy more than $1 \mathrm{MeV}$ opens up a new range of possibilities for a non-destructive examination when the inspected object is thick or dense. Other traditional techniques, such as X-ray, gamma ray and thermal neutron radiography, do not meet penetration capabilities of FNR in this area. Because of these distinctive features, this technique is used in different industrial applications such as security (cargo investigation for contraband such as narcotics, explosives and illicit drugs), gas/liquid flow and mixing and radiography and tomography of encapsulated heavy shielded low $\mathrm{Z}$ compound materials. The FNR images are produced directly during exposure as neutrons create recoil protons, which activate a scintillator screen, allowing images to be collected with a computer-controlled charge-coupled device camera. Finally, the picture can be saved on a computer for image processing. The aim of this research was to set up a portable FNR system and to test it for use in non-destructive testing of different composite materials. Experiments were carried out by using a fast portative neutron generator Thermo Scientific MP 320 .
\end{abstract}

Keywords: fast neutron $\bullet$ radiography $\bullet$ scintillator $\bullet$ recoil protons

\section{E. $\operatorname{Kam}^{凶}$}

Department of Physics

Yıldız Technical University

Esenler, Istanbul, Turkey

E-mail: erolkam@hotmail.com

\section{A. Reyhancan}

Energy Institute

Istanbul Technical University

Istanbul, Turkey

R. Biyik

Technology Development Department

TAEK

Istanbul, Turkey

Received: 4 October 2018

Accepted: 6 March 2019

\section{Introduction}

Different radiography imaging techniques such as $\mathrm{X}$-ray, gamma ray, and neutron radiography, are well-established techniques for the non-destructive testing (NDT) of materials. However, fast neutron radiography (FNR) is relatively new and is currently under development [1]. Using this technique, materials such as high-density metals, loaded plastics, cadmium, lead, tungsten, and concrete can be analysed. Compared to other techniques, fast neutrons enable NDT of thicker objects [2-5].

The FNR systems generally consist of three parts: 1. a neutron generator (must produce suitable neutron beam),

2. a converter or detector and

3. a device to record the radiation intensity.

In fast neutron radiography (FNR) systems, neutron generators are usually accelerator based and require a particle accelerator and a target [6]. A proton or deuteron beam is accelerated to the desired energy, bombarding the target material to produce fast neutrons by a nuclear reaction. In our study, a sealed deuterium-tritium (D-T) neutron source is used. Fusion of a deuterium and a tritium atom (D $+\mathrm{T}$ ) results in the creation of a He-4 ion and a neutron with a kinetic energy of nearly $14.1 \mathrm{MeV}$ [7]. 


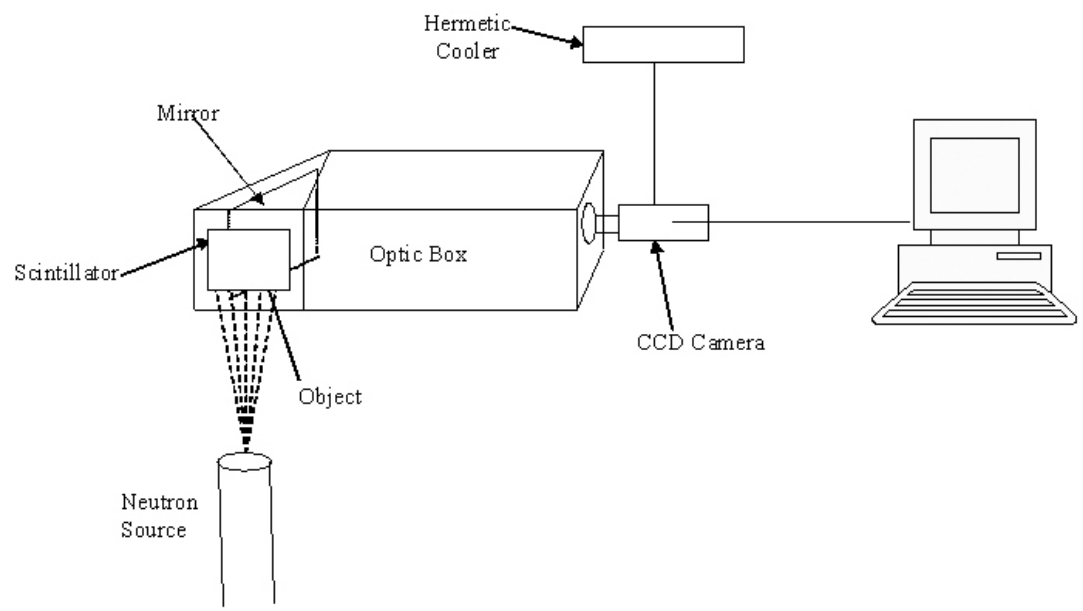

Fig. 1. Fast neutron radiography hardware.

A scintillation detector is used for FNR imaging as a converter $[3,8]$. Fast neutrons are scattered by hydrogen nuclei, and the recoil protons produced are absorbed in a scintillation plate. The scintillated product is in the visible wavelength range. Each neutron interaction with a scintillator may, therefore, be considered as a point-like light source producing a rather significant number of optical photons [9]. At FNR systems, neutron detectors work in an integrated mode, meaning individual neutrons are not counted.

In this study, we introduce a portable digital FNR system and demonstrate some images of different composite materials to show efficiency of the system. Some other parameters of the systems that are used to describe the image, such as spatial resolution, contrast sensitivity, detective quantum efficiency, dynamic range, and temporal resolution, will be discussed in another paper.

\section{Description of the system}

The FNR system is designed as shown in Fig. 1. Aluminium sheets were chosen as the construction material of the optical box because of the short half-life activation of aluminium when it interacts with fast neutrons. The inner wall of the optical box was painted matt black to prevent light reflections. The optical box system was exposed to light sources from outside to confirm that there is no light leakage inside the box. After the light leakage tests, the charge-coupled device (CCD) camera was mounted.

An accelerator-based portable neutron generator was used as a source (Thermo Scientific MP 320) (Fig. 2). The generator used D-T reactions and produced 14.1 MeV energetic neutrons with a $\sim 1 \times 10^{8}$ $\mathrm{n} / \mathrm{cm}^{2} / \mathrm{s}$ flux at $80 \mathrm{kV}$ terminal voltage and a $60-\mu \mathrm{A}$ ion beam current. In FNR systems, scintillation screens (plastic or inorganic scintillators) were used $[3,9,10]$. Because of their sensitivity to fast neutrons, inorganic $\mathrm{ZnS}(\mathrm{Ag})$ scintillators were prepared (silver-activated ZnS (30\%) of granule size $20 \mu \mathrm{m}$ dispersed in polypropylene $(70 \%)$ with dimensions $27 \mathrm{~cm} \times 27 \mathrm{~cm}$ and $2.4 \mathrm{~mm}$ in thickness). The scanning electron microscope (SEM) image of the granules and the picture of the scintillator developed by us are shown in Figs. 3a and 3b, respectively. To record the image of a composite sample material subjected

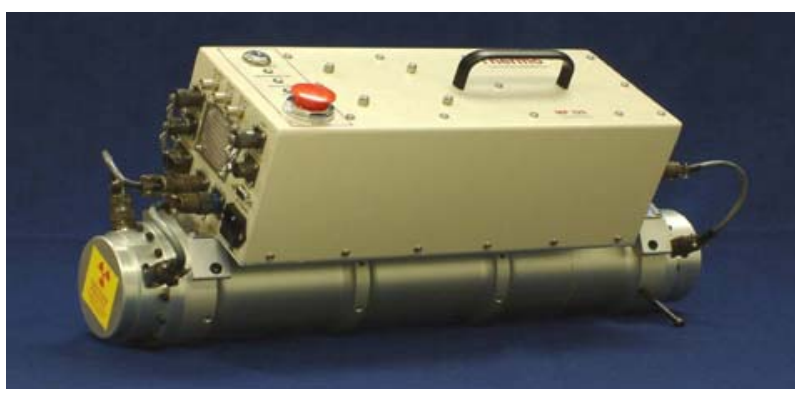

Fig. 2. Thermo Scientific MP 320 portable neutron generator.

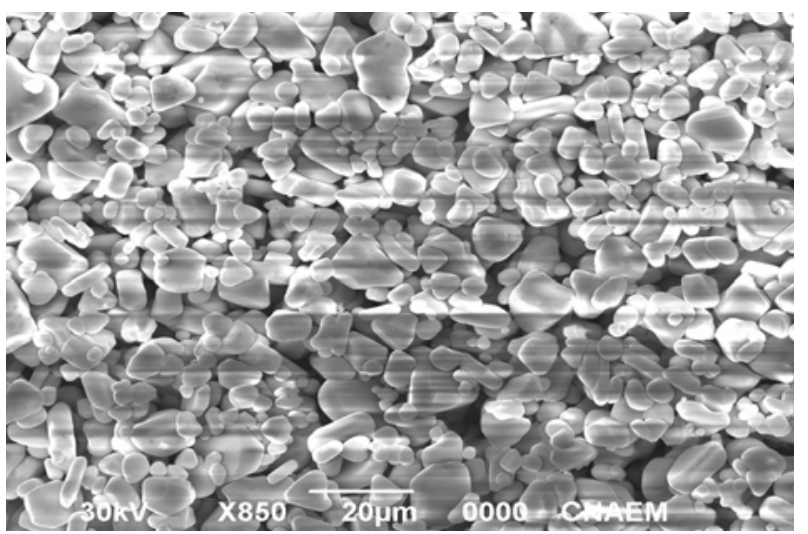

Fig. 3a. The SEM image of the $\mathrm{ZnS}(\mathrm{Ag})$ scintillator.

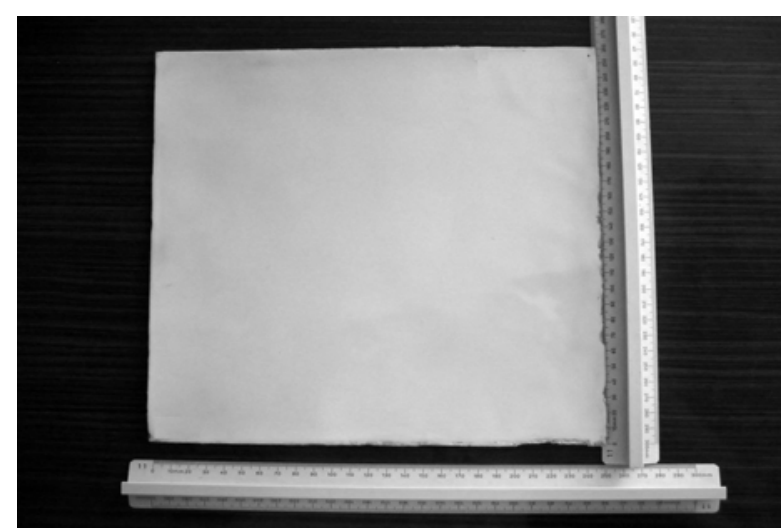

Fig. 3b. Scintillator screen $(27 \mathrm{~cm} \times 27 \mathrm{~cm})$. 
to neutrons, a scintillator, a mirror at 45 degrees, and a CCD were used as depicted in Fig. 1. The mirror was necessary to avoid direct neutron irradiation of the CCD. Furthermore, additional shielding was required to protect from stray radiation. The lens of the CCD focused light on the chip and helped to collect more light. We used Hamamatsu ORCA BT-1024G type, high resolution, $1024 \times 1024$ pixel back thinned (BT) as our high cooling performance with 4-stage peltier cooled CCD digital camera. This very high resolution, BT, back-illuminated million pixel CCD offers very high quantum efficiency of more than $90 \%$ peak and broad sensitivity from UV to NIR spectrum of 350-900 nm. Quantum efficiency is the measure of the effectiveness of an imager to produce electronic charge from incident lights. This is a particularly important property when doing low-light-level imaging.

After the desired exposure time, the obtained images need to be processed. There are a lot of software that can be obtained commercially or freely for this purpose. However, we wrote a computer software using Visual C\#, which has a simple interface and is easy to use, effective and fast. It runs only on Windows operating system and is free. A zipped pack, containing the program; some models and user manual can be obtained on request via e-mail from the authors. In our software, we used LPGL and AForge.NET libraries. The program includes common image processing units such as contrast change, grey tones, curves, brightness, threshold, filters (median, Gaussian blur, gamma correction, sharpen, adaptive smoothing, etc.) and so on.

\section{Some image examples}

Several test objects, being composed of different materials and shapes, were prepared. These include Plexiglas, plastics, aluminium, iron, copper wire and concrete. Based on the radiographic images obtained, it can be said that the spatial resolution of our system is capable of resolving objects of at least $1.5 \mathrm{~mm}$. The illustrations of the objects are given in the following.

The first tests of the imaging system were performed with a Plexiglas plate $(90 \mathrm{~mm} \times 90 \mathrm{~mm} \times$ $20 \mathrm{~mm}$ ) (Fig. 4a). The plate holes were formed with different radii and depths. The radii and depths of the holes were between 5 and $12 \mathrm{~mm}$ and 5 and $15 \mathrm{~mm}$, respectively. The numerical grey level of the Plexiglas sample was determined, and the values of the signal-to-noise ratio (SNR) are shown in Table 1. SNR is an evaluation used in science and engineering to measure how much a signal has been corrupted by noise. It is defined as the ratio of signal power to the noise power corrupting the signal. A ratio higher than 1:1 indicates more signal than noise. The higher the ratio, the less obtrusive the background noise is. As seen in Fig. 4b and Table 1, the resolution of the system is pretty good and the contrast of the holes are clearly distinguished according to their sizes.

In another test to show the response of the radiographic system against different composite

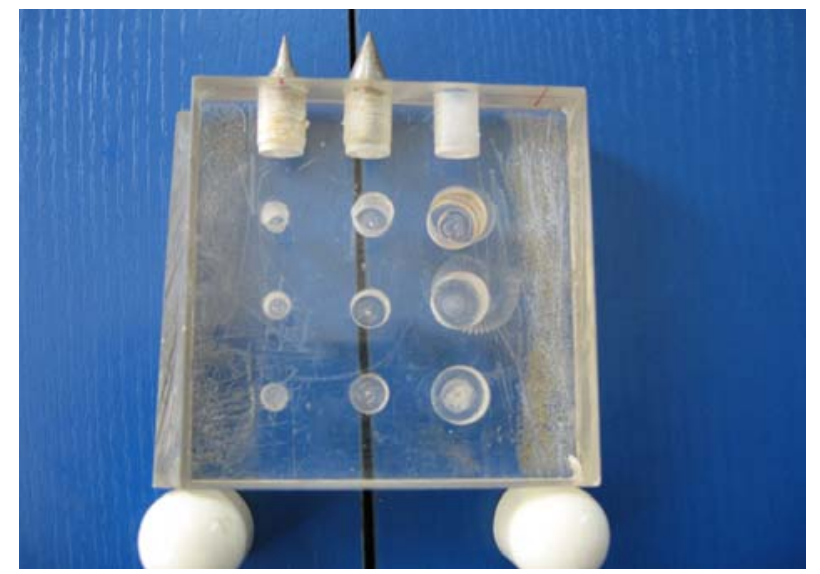

Fig. 4a. Plexiglas plate (on which holes with different radii and depths are formed).

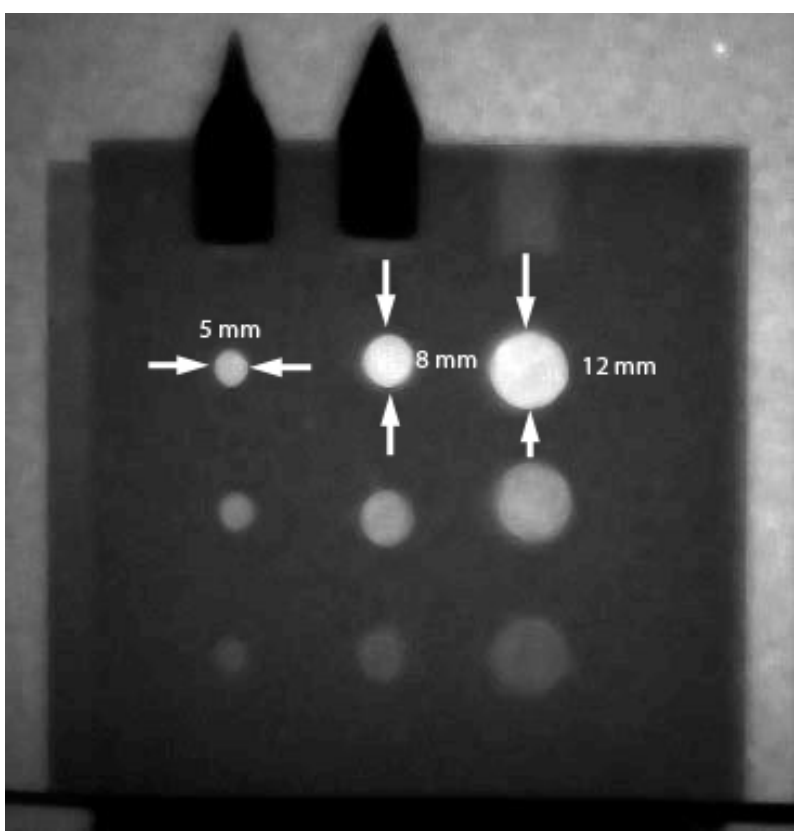

Fig. 4b. FNR image of the Plexiglas sheet.

Table 1. The values of the signal-to-noise ratio (SNR) of Plexiglas sample

\begin{tabular}{rccc}
\hline \multirow{2}{*}{$\begin{array}{c}\text { Diameter } \\
{[\mathrm{mm}]}\end{array}$} & \multicolumn{3}{c}{ Depths } \\
\cline { 2 - 4 } & $15 \mathrm{~mm}$ & $10 \mathrm{~mm}$ & $5 \mathrm{~mm}$ \\
\hline 12 & 5.31 & 3.56 & 2.32 \\
8 & 5.20 & 3.39 & 2.10 \\
5 & 5.15 & 2.97 & 1.94 \\
\hline
\end{tabular}

materials, materials with different shapes were collected together in a container. For this purpose, a cylindrical aluminium block was placed on top of an upside down half-full adhesive gum tube, and they were placed in an empty cylindrical container. Figures 5a and 5b show optical and FNR image of the materials, respectively. The FNR system not only distinguished different composite materials but also showed different contrasts on the empty side of the half-full adhesive gum.

The other example is a lantern. The FNR image showed distinctly the different grey levels of the plastic case of the lantern, the copper wire, some metals, and the lithium battery (Figs. 6a and 6b). 


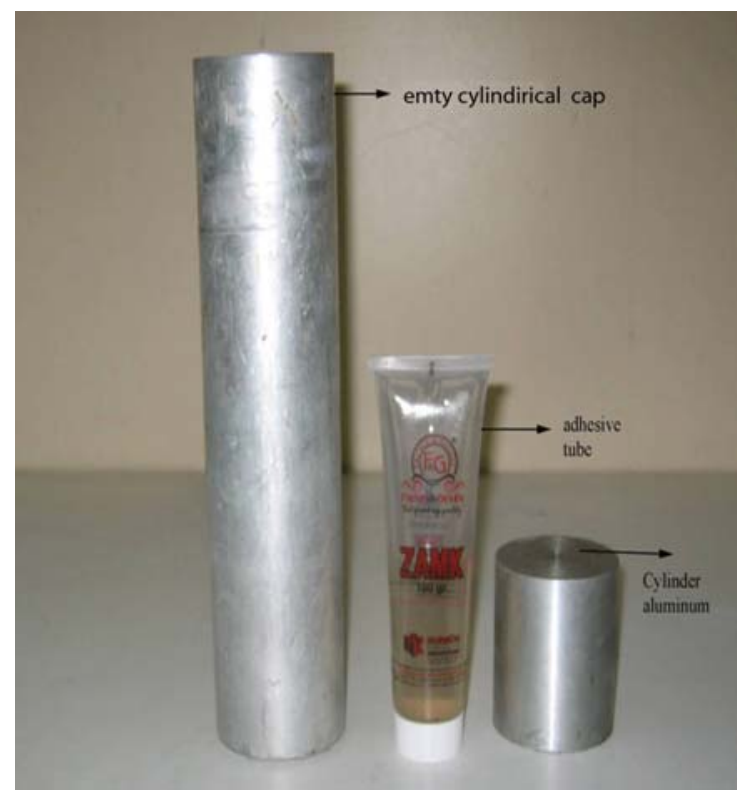

Fig. 5a. Picture of different composite materials.

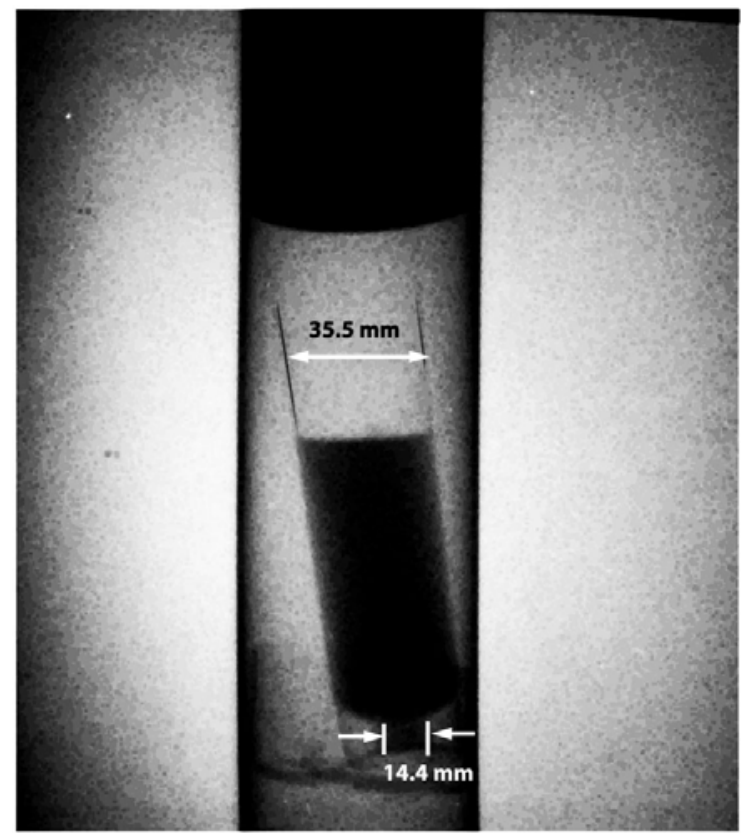

Fig. 5b. FNR images of different composite materials collected all together in a container.

The fourth image test shows the detection of iron sticks in concrete. First, moulds with two different shapes were prepared. One type consisted of steel cylindrical tubes ( $6 \mathrm{~cm}$ in radius), and the other one was a triangular wood $(30 \mathrm{~cm} \times 40 \mathrm{~cm} \times 50 \mathrm{~cm})$ (Fig. 7a). Iron sticks with different thicknesses and shapes were placed in steel moulds. Then C30-type concrete was poured. After waiting for the concrete to dry, samples were removed from the mould to take FNR images. Then, images were taken and processed. Because iron and concrete have nearly the same neutron absorption coefficients, traditional imaging methods could not distinguish the two satisfactorily. However, as depicted in Fig. 7b, three iron sticks with different shapes could easily be seen in concrete in our case. Moreover, contrast difference of curves of the iron sticks and the nut parts were

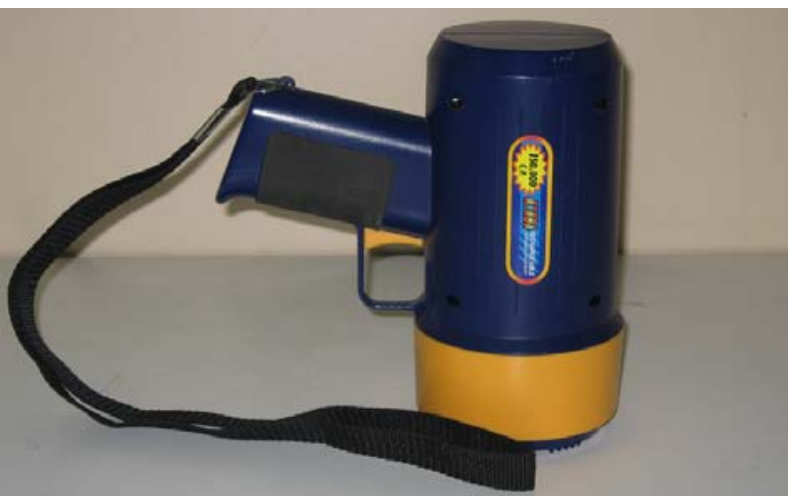

Fig. 6a. Normal lantern sample.

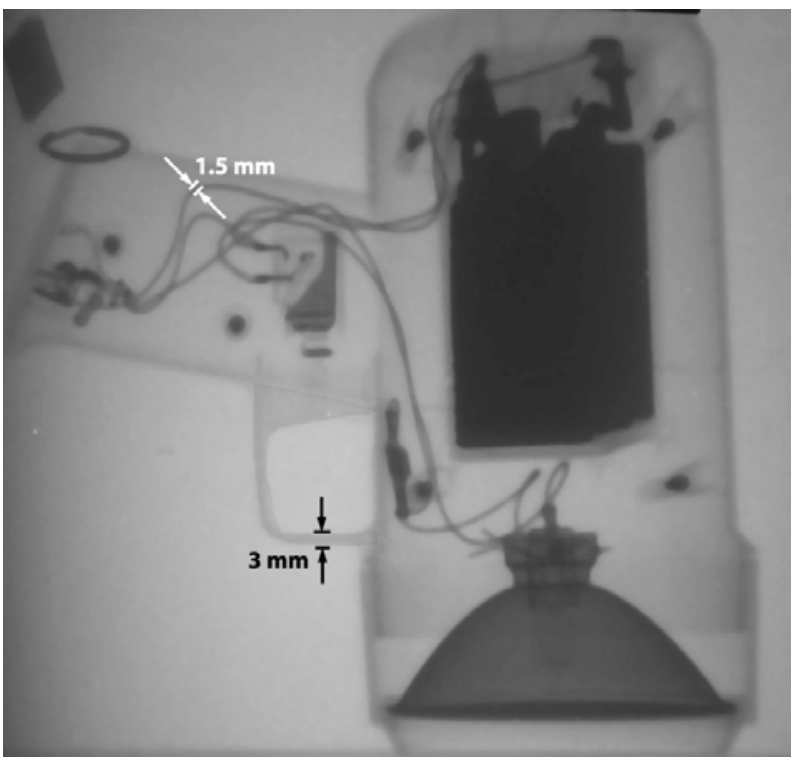

Fig. 6b. FNR image of the lantern sample.

remarkably distinguished. The image of the triangle sample also has an important value (Fig. 7c). The thickness of the triangular concrete block shown in the figure varies from $2 \mathrm{~cm}$ at the apex to $30 \mathrm{~cm}$ at the base. All the iron sticks are seen in the FNR image. Figure 7d shows grey levels of the iron sticks against pixel dimension. This result demonstrates that iron sticks and their thicknesses in concrete structures can be seen with non-destructive FNR techniques. This feature may especially be used to check the strength of historical buildings in a non-destructive way.

\section{Conclusion}

In this study, we try to show some key features of FNR systems for the industrial applications. The results obtained in this study are important in terms of demonstrating the development of FNR technique for portable use. At this point, it can be said that two basic parts of FNR need to be developed: commercially available turn-key D-T and D-D neutron generators and scintillation screens. Portable neutron sources that can be used for this purpose should be able to produce high neutron flux, and scintillation screens with high light efficiency are required.

We have presented FNR images taken for materials with different compositions, and we also showed 


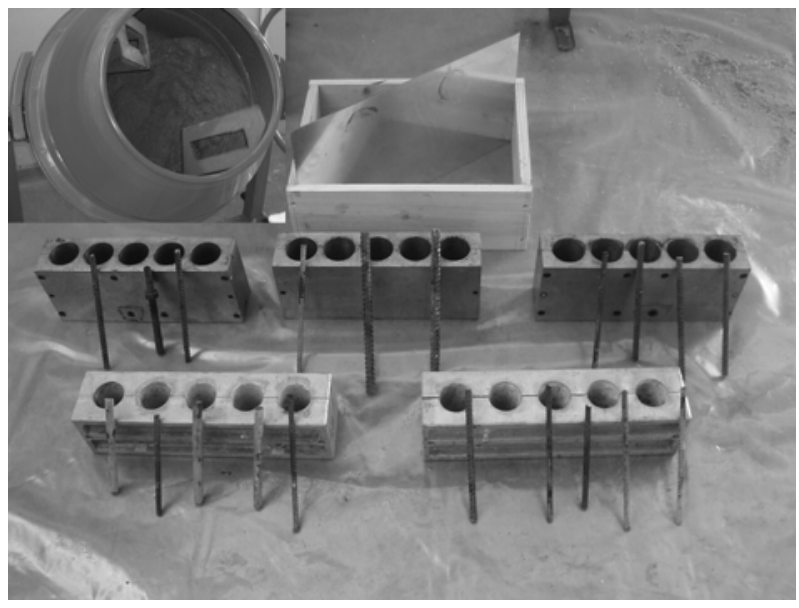

Fig. 7a. Moulds with two different shapes, cylindrical and triangular.

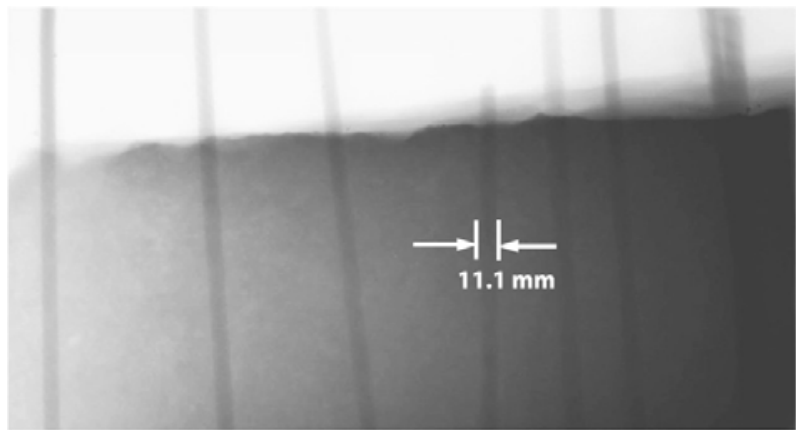

Fig. 7c. FNR images of iron sticks in a triangular concrete block.

that iron bars and thicknesses in concrete moulds can be detected using a portable neutron generator. At this point, if the work is further developed, this system can be used in the construction sector for the purpose of control and non-destructive inspection of buildings.

\section{References}

1. de Haan, V. O., van der Hagen, T. H. J. J., Federov, A., Van Veen, A., \& de Leege, P. F. A. (2005). Conceptual design of a novel high-frame-rate fast-neutron radiography facility. Nucl. Instrum. Methods Phys. Res. Sect. A-Accel. Spectrom. Dect. Assoc. Equ., 539, 321-334. doi: 10.1016/j.nima.2004.10.007.

2. Ferreira, F. J. O., Silva, A. X., \& Crispim, V. R. (2010). Electronic imaging system for neutron radiography at a low power research reactor. Radiat. Meas., 45, 806-809. doi: 10.1016/j.radmeas.2010.02.013.

3. Chankow, N., Punnachaiya, S., \& Wonglee, S. (2010). Neutron radiography imaging plate. Appl. Radiat. Isot., 68, 662-664. doi: 10.1016/j.apradiso.2009.09.21.

4. Hausladen, P. A., Bingham, P. R., Neal, J. S., Mullens, J. A., \& Mihalczo, J. T. (2007). Portable fast-neutron radiography with the nuclear materials identification system for fissile material transfers. Nucl. Instrum. Methods Phys. Res. Sect. B-Beam Interact. Mater. Atoms, 261,387-390. doi: 10.1016/j.nimb.2007.04.206.

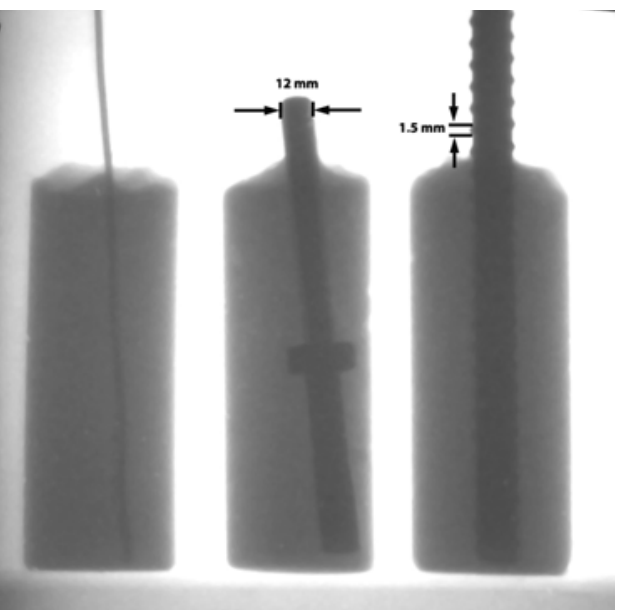

Fig. 7b. FNR images of iron sticks with different thicknesses in concrete.

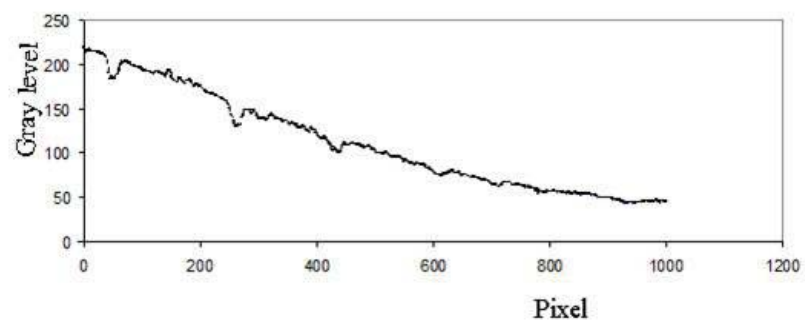

Fig. 7d. Grey levels of the iron rods against pixel dimension.

5. Fujine, S., Yoneda, K., Yoshii, K., Kamata, M., Tamaki, M., Ohkubo, K., Ikeda, Y., \& Kobayashi, H. (1999). Development of imaging techniques for fast neutron radiography in Japan. Nucl. Instrum. Methods Phys. Res. Sect. A-Accel. Spectrom. Dect. Assoc. Equ., 424, 190-199. doi: 10.1016/S0168-9002(98)01326-6.

6. Kim, K. H., Klan, R. T., \& Raju, B. B. (1999). Fast neutron radiography for composite materials evaluation and testing. Nucl. Instrum. Methods Phys. Res. Sect. A-Accel. Spectrom. Dect. Assoc. Equ., 422, 929-932. doi: 10.1016/S0168-9002(98)01048-1.

7. Popov, V., Degtiarenko, P., \& Musatov, I. (2011). New detector for use in fast neutron radiography. J. Instrum., 6, (13pp.). doi: 10.1088/1748-0221/6/01/C01029.

8. Reeder, P. L., Peurrung, A. J., Hansen, R. R., \& Stromswold, D. C. (1999). Detection of fast neutrons in a plastic scintillator using digital pulse processing to reject gammas. Nucl. Instrum. Methods Phys. Res. Sect. A-Accel. Spectrom. Dect. Assoc. Equ., 422, 84-88. doi: 10.1016/S0168-9002(98)01068-7.

9. Gongyin, Chen. (2001). Fast neutron resonance radiography for elemental imaging: Theory and applications. Unpublished Ph.D. Thesis, Massachusetts Institute of Technology.

10. International Atomic Energy Agency. (2008). Neutron imaging a non-destructive tool for materials testing. Report of a coordinated research project. Vienna: IAEA. (IAEA-TECDOC-1604). 